\title{
Analytic Study of Features for the Detection of Covert Timing Channels in Network Traffic
}

\author{
Félix Iglesias Vázquez, Robert Annessi and Tanja Zseby \\ CN Group, Institute of Telecommunications, TU Wien, Austria \\ E-mail:felix.iglesias@nt.tuwien.ac.at; robert.annessi@tuwien.ac.at; \\ tanja.zseby@nt.tuwien.ac.at
}

Received 30 November 2017; Accepted 3 December 2017;

Publication 18 December 2017

\begin{abstract}
Covert timing channels are security threats that have concerned the expert community from the beginnings of secure computer networks. In this paper we explore the nature of covert timing channels by studying the behavior of a selection of features used for their detection. Insights are obtained from experimental studies based on ten covert timing channels techniques published in the literature, which include popular and novel approaches. The study digs into the shapes of flows containing covert timing channels from a statistical perspective as well as using supervised and unsupervised machine learning algorithms. Our experiments reveal which features are recommended for building detection methods and draw meaningful representations to understand the problem space. Covert timing channels show high histogramdistance based outlierness, but insufficient to clearly discriminate them from normal traffic. On the other hand, traffic features do show dependencies that allow separating subspaces and facilitate the identification of covert timing channels. The conducted study shows the detection difficulties due to the high shape variability of normal traffic and suggests the implementation of semi-supervised techniques to develop accurate and reliable detectors.
\end{abstract}

Keywords: Covert timing channels, Network traffic analysis, Classification, Anomaly detection, Feature selection.

Journal of Cyber Security, Vol. 6_3, 245-270.

doi: $10.13052 / \mathrm{jcsm} 2245-1439.632$

This is an Open Access publication. (c) 2017 the Author(s). All rights reserved. 


\section{Introduction}

Covert channels are parasitic communication channels, which are exploited to hide the existence of actual communications but for the senders and the receivers of the covert information. They are parasitic because they are built on top of other systems, usually related to technology or information transmission means. In the case of TCP/IP protocols, covert channels use either TCP/IP header fields or time properties of network traffic packets to hide the secret message.

The existence of covert channels is usually-but not necessarily-related to illegal and criminal activities, e.g., data leakage, malware propagation, penetration attacks. One example is the network attacks during December 2015 on Ukraine power companies, whose computers were infected with Gcat. The Gcat malware created covert channels on Gmail applications to conceal $\mathrm{C} \& \mathrm{C}$ (command-and-control) operations and to pass unperceived by security systems [8]. Covert channels are a serious problem related to security and system vulnerabilities; not in vain, they were already identified as a security threat for communication networks almost 40 years ago [25].

This work focuses on covert channels over TCP/IP and, specifically, on covert timing channels, i.e., whenever time properties of TCP/IP transmissions are exploited. We continue the investigations started with [12], where covert channels were classified by studying the implications and challenges faced from the detection side. Additionally, in [12] a general solution for covert channel identification called DAT (from Descriptive Analytics of Traffic) is proposed. The DAT methodology is based on representing traffic flows with a set of statistical measurements and estimations. In [13] and [15] the scope was reduced to timing channels, analyzing and testing the detection with supervised classification in [13] and with unsupervised algorithms in [15]. Even in spite of the fact that covert timing channels are anomalies from a semantic understanding, from the perspective of statistical properties they remain in zones also occupied by normal traffic, yet they can be differentiated by supervised analysis (as shown in [15]). In other words, the separation boundaries between traffic flows with and without covert timing channels are not located in low density areas of the problem space (at least when analyzing the spaces created by the features studied in [15]).

In this paper, we extend the experiments and improve the cited research in $[12,13,15]$ as follows:

\section{Study of new features for randomness and predictability}

The suitability of five new coefficients related to the estimation of randomness and predictability in time series is studied. The addition of new 
features to the basic DAT vector format increases computational costs but is expected to enhance detection performance, being therefore justified in environments that demand high security and deal with assumable traffic volumes.

2. Validation with novel techniques

A validation phase was added to the experiments. In the validation phase, in addition to the eight covert timing techniques used in previous experiments, detectors are evaluated versus two new covert timing channels published in 2017, which are not present during training and testing phases. The robustness of statistical detection frameworks is therefore here tested as well as the hypothesis that suggests that different covert timing techniques are prone to similarly exploit the timing capacities of network traffic (within the scope of the features under test).

The rest of the paper is organized as follows: Section 2 briefly describes the covert timing channel techniques used in the experiments. Section 3 describes the features under study. Section 4 depicts the experimental design and Section 5 discusses the obtained results. Finally, conclusions are drawn in Section 6.

\section{Explored Covert Timing Channel Techniques}

For the experiments we have selected ten covert timing channel techniques. Eight of them are popular in the field of covert channel detection, widely described in [13] and in the original papers (we provide the corresponding references). The last two techniques have been published recently in [7]. We use them here for validation and testing detectors against unknown/novel techniques. We abbreviate technique names with three capital letters (or two and one number) that refer to the authors who published them. Techniques are briefly described later in this section, and Table 1 lists the specific parameters used for the generation of covert channels.

For a better understanding of the developed methods, it is important to clarify two different terms related to time properties. The Inter Arrival Time (IAT) refers to the time between packets seen from the perspective of the receiver; on the other side, the Inter Departure Time (IDT) is the time between packets seen from the perspective of the sender. Synthetically expressed: IAT = IDT + tx_delay, where "tx delay" is the transmission delay. 


\subsection{Packet Presence (CAB)}

In this technique communication partners are time synchronized and agree on a predefined time interval $t_{\text {int }}$. The presence or absence of a packet within the predefined time interval stands for the covert symbol 1 or 0 respectively [4].

\subsection{Differential/Derivative (ZAN)}

This is a technique originally described for the Time-to-Live (TTL) field [33], yet easily applicable to IATs. This technique makes use of two parameters, $t_{b}$ and $t_{\text {inc }}$. $t_{b}$ refers to the base IDT at which packets may be sent and $t_{i n c}$ to the time that may be added or substracted from $t_{b}$. If the covert symbol 0 is to be transmitted, the IDT is set to $t_{b}$; if the covert symbol 1 is to be transmitted, however, the IDT is set to $t_{b} \pm t_{\text {inc }}$. Addition and substraction are applied alternately on subsequently occurring 1 symbols.

\subsection{Fixed Intervals (BER)}

This straightforward technique [1] agrees on two different IDTs to mask binary symbols. For instance, $t_{0}$ for 0 and $t_{1}$ for 1 .

\subsection{Jitterbug/Modulus (SHA)}

The technique [28] is designed to interfere legitimate communications. It uses a base sample interval $\omega$ and adds some delay to IDTs. A covert 1 or a 0 is interpreted depending on if a given IAT is divisible by $\omega$ or only by $\omega / 2$.

\subsection{Huffman Coding (JIN)}

By using Huffman coding, every covert symbol is encoded in a set of packets with different IDTs [32]. The proposed codification tries to optimize communication bandwidth based on the frequency of ASCII symbols observed in English texts. The given implementation only covers a set of basic lower case characters and numbers.

\subsection{One Threshold (GAS)}

This technique [9] uses a time threshold $t_{h}$ to discriminate $0 \mathrm{~s}$ and $1 \mathrm{~s}$. If a given IAT is above $t_{h}$ it will mark 1,0 if below. For the implementation we used additional times to create the covert channel and match the proposed rule (Section 1). 


\subsection{Packet Bursts (LUO)}

In this technique [20] packets are sent in bursts. The number of packets sent per burst marks the intended covert symbol to transmit. Between two subsequent bursts, some time $t_{w}$ is waited. In principle, this technique is not exploited for binary channels, but manages some symbols (few, about 10 or 16).

\subsection{TCP Timestamp Manipulation (GIF)}

This technique [10] uses TCP timestamps to convey covert information. Packet IDTs are altered in such a way that the least significant bit (LSB) of the TCP timestamp matches the covert information. TCP timestamps are updated at a specific clock frequency, usually 100, 250, or $1000 \mathrm{~Hz}$. For this reason, this technique establishes a waiting $t_{b}$ between consecutive packets (authors suggest a minimum of $10 \mathrm{~ms}$ ). If the LSB is the same as the desired numerical covert symbol, the packet is sent; if not, the sender checks the LSB again after a defined $t_{w}$.

\subsection{ASCII Binary Encoding (ED1)}

This technique [7] encodes the covert symbol 0 with a waiting time $t_{0}$ (authors suggest $300 \mathrm{~ms}$ ), whereas the covert symbol 1 triggers the immediate sending of a packet. Note that $t_{0}$ is not defining any IDT; therefore, sending 0 s does not imply sending any packet. For example, if 'A', which corresponds to the ASCII encoding ' 01000001 ', is to be covertly send, the sender will follow this sequence: (1) wait $300 \mathrm{~ms}$, (2) send one packet, (3) wait $1.5 \mathrm{~s} \mathrm{(300} \mathrm{ms} \times 5$ ), (4) send one packet. Messages are forced to finish transmitting a ' 1 '.

\subsection{5-Delay Encoding (ED2)}

This technique [7] encodes every letter of the English alphabet with a 5digit numerical code where each digit corresponds to a different IDT. Authors propose a waiting time between covert letters, $t_{w}$.

\section{Selected Features}

As mentioned in Section 1, a recent classification of covert channels is presented in [12]. Moreover, DAT is introduced as a general methodology to identify covert channels in network traffic flows. The DAT approach is fundamentally based on a set of statistical figures and estimations extracted from TCP/IP flow header fields as well as IATs. The specific field under 
study - IAT for the case of covert timing channels-is initially tackled from a time series analysis perspective. Further derived studies that focus on covert timing channels are developed in [13] and [15]. Here we explore the features used in these three cited works $([12,13]$ and [15]) and add five new features related to regularity and randomness in time and symbol series.

Before depicting features, it is worth remembering that detectors must define two time parameters to enable time series analysis:

- Sampling time, which states the desired binning (time resolution, or granularity) for the time series analysis. DAT detectors and the experiments conducted here work with a 1-millisecond sampling time by default.

- Observation period, which establishes the maximum time-window for the observation of a given flow. DAT detectors and the experiments conducted here work with a 5-minute observation period by default. The feature vector extracted from IATs of analyzed flows is:

$$
\text { flow_vec }=\left\{U, S_{k}, \mu_{\omega S}, S_{s}, p(M o), \rho_{A}, H_{a}, T_{R}, T_{S}, H_{q}, K, p k t s\right\}
$$

The meaning of each feature is described below.

\subsection{Simple Statistical Figures}

- $\boldsymbol{U}$ - number of unique values. $U$ stores the number of non-repeated IAT values observed in the analyzed flow.

- pkts - total number of packets in the flow. pkts is simply a counter that contains the total number of packets observed in the analyzed flow.

- $\boldsymbol{p}(\mathbf{M o})$ - Mode frequency. $p(\mathrm{Mo})$ is a percentage value calculated as the quotient between the number of IAT Mode value repetitions and the total observed IATs in the flow (i.e., pkts - 1).

- $c$ - estimation of potential covert byte-equivalent symbol. The calculation of $c$ is based on empirical tables and tries to estimate the number of potential covert bytes sent in a flow [12]. It uses $S_{k}$ (defined in Section 3.2) to guess the number of different symbols that the covert channel might be applying. For example, if $S_{k}$ reveals a possible binary channel, $c$ becomes: $c=(p k t s-1) / 7$, assuming that 7 IATs values are necessary to transmit a covert byte-equivalent symbol ${ }^{1}$.

\footnotetext{
${ }^{1}$ It might be surprising the use of " 7 " instead of " 8 " in the quotient for estimating byteequivalent symbols. The $c$ index is an inflated estimation of the transmitted covert information and assumes that it consists of text. In this respect, note that ASCII most relevant/used symbols are less than $2^{7}=128$.
} 
An example should help to understand the introduced features. For instance, given the following sequence of IATs in a flow $i$ :

$$
\operatorname{IAT}_{i}=\{5,15,14,4,5,6,16,15,5,15,14,15,4,5\} \mathrm{ms}
$$

Feature values would be: $U=6$, i.e., the cardinality of the unique values set: $\{4,5,6,14,15,16\} ; p(M o)=4 / 14=0.29$, given that the Mode (15) occurs 4 times; $p k t s=15$; and $c=14 / 7=2$, since $S_{k}=2$.

\subsection{Multimodality Estimation}

Multimodality refers to values that either significantly occur more often than others in the series or appear as attractors in the series distribution. Estimating multimodality means providing the number of such attractors. The features related to multimodality are:

- $\boldsymbol{S}_{\boldsymbol{k}}$ - multimodality based on kernel density estimations. $S_{k}$ is the number of peaks of the curve that approximates the empirical distribution of IATs by using a gaussian kernel. Figure 1 shows two examples with similar estimated curves and both with $S_{k}=2$. Kernel density estimation for assessing multimodality is widely described in [29], and their application for covert channels is discussed in [12].

- $\boldsymbol{S}_{\boldsymbol{s}}$ - multimodality based on pareto analysis. $S_{s}$ is the number of outstanding values that appear in the IAT histogram. It follows the principles of Pareto analysis [24] and the specific calculation is depicted in [12]. In short, it is the number of values that show a considerable high frequency compared to the rest of the histogram. Figure 1 shows two examples: in the left one $S_{s}=2$, whereas in the right plot $S_{s}=6$. $S_{s}$ is expected to be irrelevant for the case of IATs as-considering a millisecond resolution-IAT series distributions tend to look like distributions of continuous variables.

- $\boldsymbol{\mu}_{\boldsymbol{\omega} S}$ - average distribution width. $\mu_{\omega S}$ is the mean of the characteristic standard deviations of the gaussians drawn by the kernel density estimations. More intuitively: it captures the average peak width of the mountains that appear in the empirical distribution.

\subsection{Regularity/predictability Estimators}

The coefficients described in this section try to provide an estimation of the randomness, the unpredictability or the regularity of a time series. $\rho_{A}$ was 

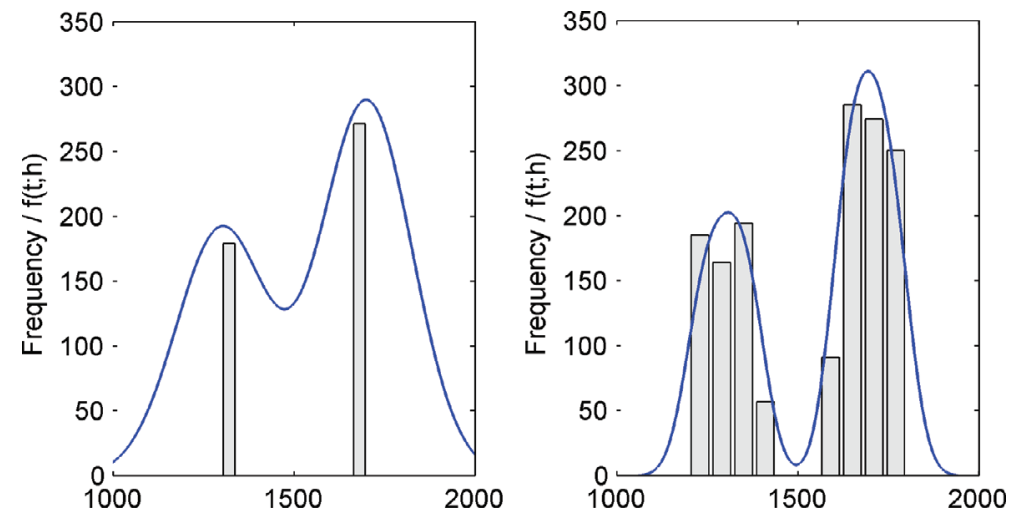

Figure 1 Histograms (bars) and kernel density estimations (curves) of two different numerical series. Left plot: $S_{k}=2, S_{s}=2$. Right plot: $S_{k}=2, S_{s}=6$. Density magnitudes are omitted as only the number of peaks is relevant for the purpose of the estimation. Figure taken from [12].

initially proposed in [12], whereas the other five measurements are added here to test if their inclusion enhances detector performances. In addition to the associated computational costs, the main drawback of all these coefficients is that they require time series be long enough (the minimum threshold is approximately placed between 10 and 100 elements). For short time series, coefficient values are either meaningless or not computable. This fact does not imply a serious problem for covert timing channel detection as, by default, short flows-specially in binary covert channels-cannot contain significant amounts of information and can be directly discarded by the detector. For instance, a binary channel that hides the sentence "hello world!" as 7-bit ASCII characters requires $1+12 \times 7=85$ packets (84 IATs).

- $\rho_{A}$ - sum of autocorrelation coefficients. $\rho_{A}$ is the sum of autocorrelation coefficients, i.e., the IAT time series is paired with delayed versions of itself and correlation coefficients are extracted. Later, such coefficients are summed and a final value is obtained. In short, $\rho_{A}$ tries to unmask repetition patterns that fit the studied delays. Values close to 1 disclose the existence of patterns and regularity.

- $\boldsymbol{T}_{\boldsymbol{R}}$ - runs test. The runs test [2] checks if it is reasonable to consider that each element in the studied time series is independent and originates from the same distribution. For a non-binary time series $A$, an intermediate step is required:

$$
B=A-\mu_{A}
$$


where $\mu_{A}$ is the statistical mean of $A$. $R$ is the runs of $B$, i.e., the number of sub-series of negative or positive values within $B$. Finally,

$$
Z=\frac{R-E[R]}{S[R]}
$$

where $E[R]$ is the expected number of runs, and $S[R]$ stands for the expected standard deviation of $R$. Finally,

$$
T_{R}=|Z|-Z_{1-\alpha / 2}
$$

For time series with more than 20 elements, they are considered nonrandom if $T_{R}$ is positive. ${ }^{2}$ This test is widely explained in [2].

- $\boldsymbol{T}_{\boldsymbol{S}}$ - sign test. The sign test [21] is similar to the run test. Given the time series $A$, instead of counting runs, it takes consecutive pairs of values and constructs a new time series as follows:

$$
B=A_{2}-A_{1}, A_{3}-A_{2}, \ldots, A_{n}-A_{n-1}
$$

being $n$ the total number of elements in $A$. $P$ is the signs of $B$, i.e., the number of positive elements in $B$. Therefore,

$$
Z=\frac{P-E[P]}{S[P]}
$$

where $E[P]$ is the expected number of signs, and $S[R]$ stands for the expected standard deviation of $R . T_{S}$ is defined similarly to $T_{R}$ :

$$
T_{S}=[Z]-Z_{1-\alpha / 2}
$$

where time series with more than 20 elements are considered non-random if $T_{S}$ is positive.

- $\boldsymbol{K}$ - Kolmogorov complexity or compressibility. Given a string, the Kolmogorov complexity is defined as the length of the shortest computer program that generates such string [18]. The calculation of the Kolmogorov complexity, as originally defined, presents problems related to computability. Nevertherless, considering long-enough strings, using lossless compression for approximating Kolmogorov complexity has proven to satisfactorily estimate upper bounds in different domains [19]. In our experiments, we approximate $K$ as:

$$
K=\operatorname{len}(B) / \operatorname{len}(A)
$$

${ }^{2}$ For a $5 \%$ significance level, $\mathrm{Z}_{1-\alpha / 2}=1.96$. 
with len $(A)$ being the length of the time series $A$ and len $(B)$ the length of the compressed version of $\mathrm{A}$ by $z l i b^{3}$, i.e., $B=\operatorname{zlib}(A)$.

- $\boldsymbol{H}_{\boldsymbol{q}}-$ Hurst exponent. The Hurst exponent is strongly linked to the fractal properties of a sequence of values or series, therefore it also provides a measure of autocorrelation and self-similarity. In the case of time series, it gives a quantitative estimation of the trend to regress to the mean value or to be inclined to move in a certain direction [17]. Values between $0.0<H_{q}<0.5$ and $0.5<H_{q}<1.0$ stand respectively for negative and positive autocorrelations, while $H_{q}=0.5$ corresponds to uncorrelated Brownian processes [6]. ${ }^{4}$

- $\boldsymbol{H}_{\boldsymbol{a}}$ - Approximate Entropy. Approximate entropy is a statistical method devised to measure complexity and regularity of a system, which has proven to be suitable for short (but more than 100 data points) and noisy time-series [27]. ${ }^{5} H_{a}$ reflects the existence of patterns in a series that makes future values more predictable. Small $H_{a}$ values tending to 0 are expected for time series that contain repetitive patterns, whereas high values are expected for chaotic behaviours.

As an example, Figure 2 shows two time series: T1, generated at random, and $\mathrm{T} 2$, exhibiting a clear pattern. Regularity/predictability coefficients are shown below the figure.

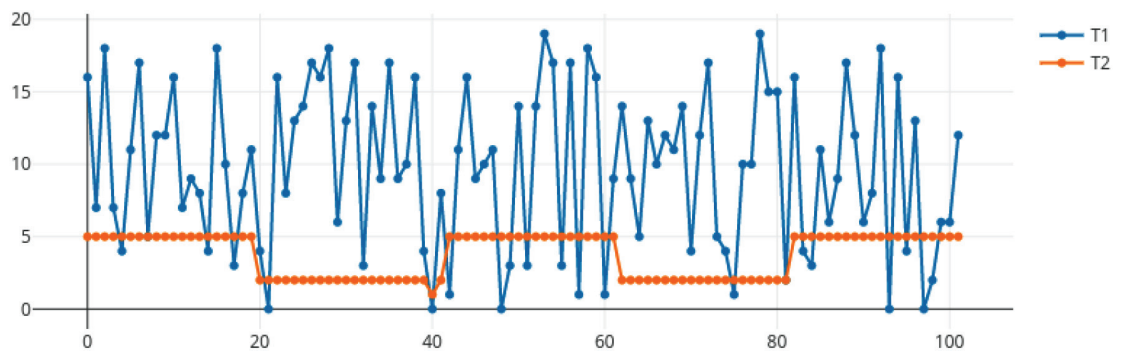

Figure 2 Regularity/predictability coefficients for two example time series.

$\mathrm{T} 1: \rho_{A_{1.5}}=0.73, T_{R}=-1.76, T_{S}=-1.61, K=1.00, H_{q}=0.68, H_{a}=2.32$.

$\mathrm{T} 2: \rho_{A_{1.5}}=0.09, T_{R}=7.49, T_{S}=14.66, \quad K=0.31, H_{q}=0.71, H_{a}=0.08$.

\footnotetext{
${ }^{3}$ https://zlib.net/

${ }^{4}$ For the experiments we have used the hurst implementation from Python library nolds 0.3.4: https://pypi.python.org/pypi/nolds

${ }^{5}$ For the experiments we have used the approximate entropy implementation from Python library nolds 0.3 .4 : https://pypi.python.org/pypi/nolds
} 


\section{Design of Experiments}

The experiments conducted in this research use real data flows for the traffic free of covert channels (henceforth called overt datasets) and flows with covert channels created by an ad-hoc traffic generation framework (henceforth called overt datasets). We describe them in this section.

As introduced in Section 3, flows are tracked 5 minutes as longest (observation period), and the sampling time for the IAT data is fixed to 1 millisecond. Final preprocessed datasets for experiment replication and further algorithm testing are publicly available to download from our webpage in [31].

\subsection{Overt Datasets}

Real traffic - in principle assumed free of covert channels - has been obtained from the MAWI Working Group Traffic Archive ${ }^{6}$. The MAWI project promotes network traffic research by daily publishing 15 minutes of TCP/IP backbone traces, from 2:00 pm to 2:15 pm GMT. MAWI datasets are anonymized and do not contain payload. We used captures from three days in 2017: a) January 31, b) February 28, and c) March 31, randomly selecting flows to create three overt datasets, namely: nocc_training, nocc_testing and nocc_ validation.

Since, for the case of covert timing channels, DAT detectors automatically consider flows with less than 10 packets as overt flows (i.e., they are too short to contain a covert timing channel), all flows with less than 10 packets were removed from the overt dataset. In [13] and [15] flows with less than two packets were removed instead. This difference makes current experiments more demanding and challenging as covert flows are only compared with overt flows that potentially look like covert flows. Performance indices are expected to be worse due to this reason. It is important to remark here that datasets used in the experiments do not try to be representative of real traffic conditions (were the rate of covert channels is absolute negligible), but submit the analysis to problem spaces that are suitable for the knowledge extraction and feature testing.

After preprocessing steps, overt datasets contain 30000 flows in nocc_training, 30000 flows in nocc_testing and 46193 in nocc_validation.

\footnotetext{
${ }^{6}$ http://mawi.wide.ad.jp/mawi/
} 


\subsection{Covert Datasets}

Covert datasets were also generated in three groups, namely:

- cc_training (1022 flows), including flows generated by using CAB, BER, SHA, JIN, GAS, LUO, ZAN and GIF techniques.

- cc_testing (1036 flows), including flows generated by using CAB, BER, SHA, JIN, GAS, LUO, ZAN and GIF techniques.

- $c c_{-}$validation (1232 flows), including flows generated by using $\mathrm{CAB}$, BER, SHA, JIN, GAS, LUO, ZAN, GIF, ED1 and ED2 techniques.

Each covert dataset was generated with a different set of files to be secretly sent. Each set of files consisted of various types of data, including plain-text (text files, list of passwords, technical reports and programming scripts), images (PNG and JPEG), compressed files (in ZIP and GZ formats), and encrypted files (in 3DES and GnuPG formats). Each singular channel was generated with a different parameterization, random seed and data to be covertly sent. Parameter value ranges were defined according to the original publications (whenever provided); otherwise, they were tuned based on traffic measurement expert knowledge. We also increased some parameter values whenever the source publication did not properly consider transmission delays for large networks. Table 1 shows the value ranges used for the random generation of parameters.

Table 1 Parameters used for the generation of covert channels. Parameters randomly fell within the shown intervals (uniform random distribution)

\begin{tabular}{ll}
\hline Technique & Parameters \\
\hline tx_delay & $\begin{array}{l}\text { By default, transmissions delays are modeled with a Lomax (Pareto } \\
\text { Type II) distribution with } \alpha=3 \mathrm{~ms} \text { and } \lambda=10 \mathrm{~ms} .\end{array}$ \\
\hline CAB & $\begin{array}{l}t_{\text {int }} \in[60,140] \mathrm{ms}, \text { being } t_{\text {int }} \text { the base time window in which the } \\
\text { presence or absence of a packet sets the covert symbol. }\end{array}$ \\
\hline BER & $t_{0} \in[10,50] \mathrm{ms}, t_{1} \in[80,220] \mathrm{ms} . t_{0}$ stands for the IDT of covert 0s and \\
& $t_{1}$ for covert $1 \mathrm{~s}$. \\
\hline SHA & The ground transmission was modeled by using a Gamma distribution with \\
& $k \in[40,760] \mathrm{ms}$ and $\phi \in[40,360]$ ms. The SHA technique uses $\omega \in[10$, \\
& $90]$ ms as sampling interval to manipulate the sending with little delays. \\
\hline GAS & IDT distribution for 0-packets: $t_{0}=t h-t_{s}$ \\
& IDT distribution for 1 -packets: $t_{1}=t h-t_{s}+t_{a}, t h \in[100,300]$ ms, \\
& $t_{s} \in[60,140] \mathrm{ms}, t_{a} \in[20,80]$ ms. $t h>t_{a}+t_{a}$ \\
\hline JIN & We used the codification proposed in [32]. \\
\hline LUO & $t_{w} \in[50,250]$ ms. $t_{w}$ is the waiting time between bursts. Packets in a \\
& burst are sent every ms. \\
\hline
\end{tabular}




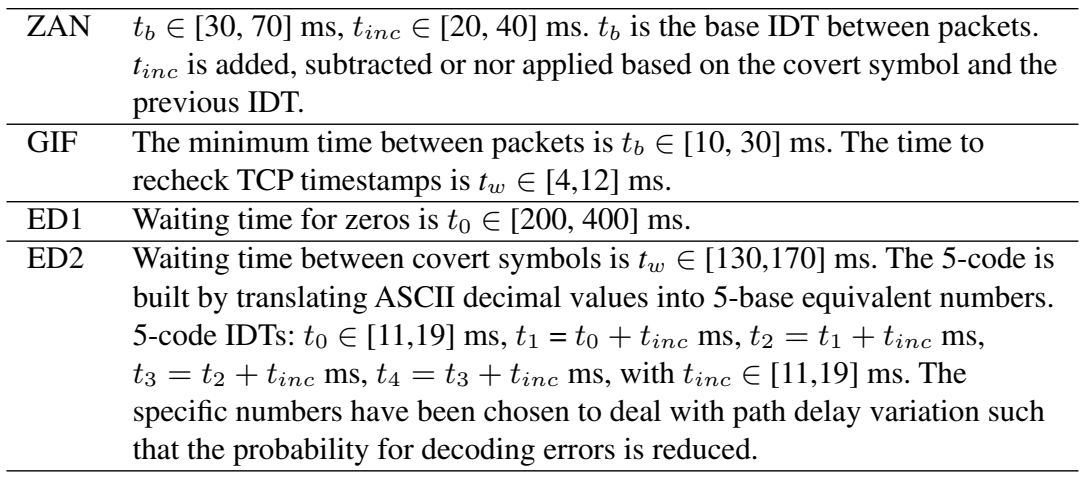

\subsection{Analysis Methods}

Covert and overt datasets were explored in sequential steps:

1. Univariate analysis and feature correlation

As a first step, features were studied separately for the $c c$ _training and nocc_training datasets by univariate analysis, aiming to detect noticeable differences in simple statistical figures. Later on, Pearson correlation between features were checked in order to detect distinct feature dependencies in overt and covert traffic.

2. Feature selection

$c c_{-}$training and nocc_training were joined in a single training dataset to study feature dependencies with regard to the binary class labels. Features were weighted by feature selection filters and hybrid schemes with different criteria. i.e., decision trees, maximum relevance, information gain, correlation and the gini index-the theoretical background of such indices can be consulted in [26] and [23]. Additionally, feature weighting methods were embedded in a subsampling structure to reinforce result robustness by means of stability selection [22]. Obtained ranks were compared and a final feature set is proposed.

3. Binary classification and validation

In this phase, training datasets are presented to learners. Obtained models are tested with testing datasets and validated with validation datasets, which explore the effect of including non-trained covert timing channels in the analysis. The training is performed with 10 -fold cross-validation, with stratified sampling to keep label proportions in each validation fold. Experiments are repeated with different feature sets to evaluate the results of the feature selection phase. Used learning schemes are: decision trees, 
random forests, SVMs, neural networks, Bayes-based ensembles and k-Nearest neighbors classifiers.

\section{Unsupervised analysis}

Training data is also analyzed by unsupervised outlier detection algorithms in order to see if the new proposed features make covert flows be outliers. Experiments slightly differ from the ones conducted in [15], where overt and covert datasets where presented together to the algorithms. Such experimental scheme might break the normal environment where covert channels appear by overpopulating the space with covert samples (in [15] they consisted on about 5\% of the total flows). Covert channels are expected to be much more infrequent among overt data. Therefore, here, outlier detection models are calculated without covert channels, and covert flows are later contrasted with the model and ranked one by one, i.e., every covert flow is isolated and independently compared with the whole overt dataset. Used algorithms are: LOF [3], COF [30], INFLO [16] and HBOS [11]. Used performance indices are: $P @ n$, precision at the top n ranks; Adj.P@n, adjusted P@n; $A P$, average precision; Adj.AP, adjusted AP; $M a x F 1$, Maximum F1 score; Adj.MaxF1, adjusted MaxF1 score; ROC/AUC, area under the ROC curve. Explanations of such indices can be consulted in [5].

\section{Results}

The results of the experiments described in Section 4.3 are shown and discussed in this section.

\subsection{Univariate Analysis and Feature Correlation}

Correlation analysis shows some linear dependencies among the studied features in overt traffic (Figure 3, left plot). Even thought it obviously depends on the features under observation, the characteristics of network traffic make finding high-related features a common situation, as observed in [14]. The positive correlation between the number of $U$ (i.e., unique IATs values) and $S_{k}$ (i.e., distribution-based multimodes) is not surprising, as well as the correlation between pkts and $c, T_{R}$ or $T_{S}$, which are expected to be higher as the number of packets increase. A special attention deserves indices among the regularity estimators. Entropy measures $H_{a}$ show inverse correlation with $U$, which makes sense according to the definition of entropy. The Kolmogorov coefficient $K$ shows inverse correlation with $p(M o)$ and $T_{S}$, which also makes sense because $K$ inversely defines chaos or irregularity if compared to $p(M o)$ or $T_{S}$. 

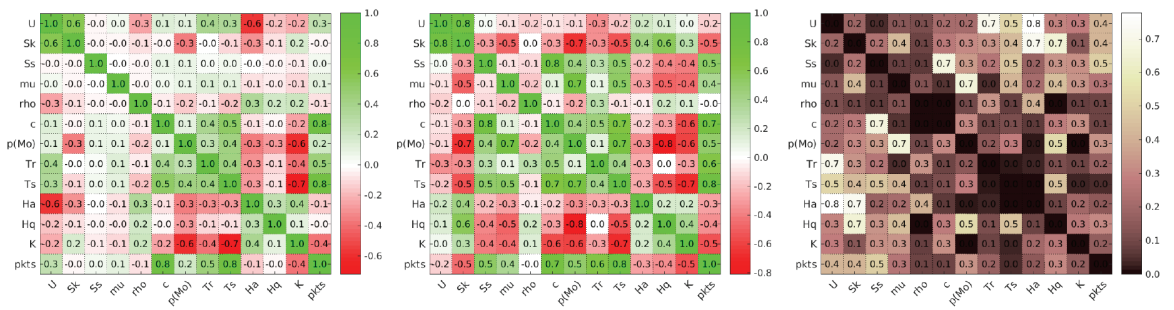

Figure 3 Results of the correlation analysis. Left matrix $(A)$ : correlations in the overt dataset. Middle matrix $(B)$ : correlations in the covert dataset. Right matrix $(C)$ : absolute differences between the correlations in the overt and the covert data set, i.e., $c_{i, j}=\left|a_{i, j}-b_{i, j}\right| \forall i, j$ being $i$ and $j$ indices for rows and columns, and $a_{i, j}, b_{i, j}, c_{i, j}$ elements of $A, B, C$ respectively.

The plot in the middle of Figure 3 shows the case of the covert dataset. Covert traffic shows more extreme correlation indices. This fact suggests that covert flows follow more strict and regular structures with respect to the selected features. Another way to look at this picture is acknowledging that overt traffic is richer in shapes and possibilities. Such results foresee a classification scenario where covert flows can be discriminated, but false positives might be also likely. The right plot in Figure 3 shows which features - high values, light backgrounds - might be potentially determinant to establish classification boundaries to separate overt and covert traffic.

The comparison between univariate analysis of features for overt and covert datasets reveal significant differences in the central tendency measures (Table 2). However, values that significantly differ from overt to covert traffic also show high dynamic ranges (represented by the standard deviation).

Table 2 Univariate statistics for overt and covert traffic. Since feature distributions are not Gaussians, we use nonparametric Confidence Intervals (approximately 95\%) over the median

\begin{tabular}{|c|c|c|c|c|c|c|c|c|c|c|}
\hline & \multicolumn{5}{|c|}{ Overt Traffic } & \multicolumn{5}{|c|}{ Covert Traffic } \\
\hline & mean & stdev & median & CI_low & CI_high & mean & stdev & median & CI_low & CI_high \\
\hline$u$ & 28.75 & 38.73 & 15.00 & 15.00 & 15.00 & 196.10 & 96.17 & 159.00 & 155.00 & 165.00 \\
\hline$S_{k}$ & 18.83 & 28.88 & 11.00 & 11.00 & 11.00 & 168.38 & 114.49 & 139.00 & 132.00 & 146.00 \\
\hline$S_{s}$ & 2.33 & 6.61 & 2.00 & 2.00 & 2.00 & 2.62 & 2.16 & 2.00 & 2.00 & 2.00 \\
\hline$\mu_{\omega S}$ & 0.01 & 0.05 & 0.00 & 0.00 & 0.00 & 0.02 & 0.04 & 0.01 & 0.01 & 0.01 \\
\hline$\rho_{A}$ & 0.11 & 0.08 & 0.10 & 0.10 & 0.10 & 0.04 & 0.03 & 0.04 & 0.03 & 0.04 \\
\hline$c$ & 88.53 & 1462.21 & 3.29 & 3.29 & 3.29 & 781.63 & 2139.75 & 289.36 & 246.71 & 351.00 \\
\hline$p(M o)$ & 0.27 & 0.22 & 0.20 & 0.20 & 0.20 & 0.07 & 0.07 & 0.05 & 0.05 & 0.05 \\
\hline$T_{R}$ & -0.17 & 3.57 & -1.05 & -1.07 & -1.02 & 1.36 & 4.57 & -0.39 & -0.50 & -0.22 \\
\hline$T_{S}$ & 3.21 & 18.86 & 0.00 & 0.00 & 0.00 & 1.87 & 4.71 & 0.29 & 0.10 & 0.50 \\
\hline$H_{a}$ & 6.95 & 4.43 & 10.00 & 10.00 & 10.00 & 0.57 & 0.06 & 0.58 & 0.58 & 0.58 \\
\hline$H_{q}$ & 129.77 & 335.41 & 0.41 & 0.41 & 0.41 & 1.20 & 0.44 & 1.29 & 1.26 & 1.31 \\
\hline$K^{2}$ & 0.97 & 0.12 & 1.00 & 1.00 & 1.00 & 1.00 & 0.02 & 1.00 & 1.00 & 1.00 \\
\hline pkts & 414.49 & 4014.59 & 23.00 & 22.00 & 23.00 & 3647.22 & 4465.61 & 1774.00 & 1615.00 & 2062.00 \\
\hline
\end{tabular}


This fact anticipates overlapping areas in the problem space that can hinder accurate classification.

In short, univariate analysis and correlation tests reveal:

- Overt and covert traffic show different central tendencies and featurecorrelation profiles. This is an evidence of the existence of patterns that can be learned by classification schemes.

- Some feature pairs-specially $\left[U, H_{\alpha}\right],\left[U, T_{R}\right],\left[S_{k}, H_{q}\right],\left[c, S_{s}\right]$, $\left[p(M o), \mu_{\omega S}\right]$ —show opposed correlation relationships when overt and covert traffic are compared. Such pairs might be keys that facilitate the binary classification.

- Redundancy is high and common in features, specially for covert traffic. This fact can imply difficulties for selecting the right features and for some classification techniques (e.g., naive Bayes).

\subsection{Feature Selection}

Feature selection experiments expose that features are ranked differently depending on the used feature selection method, as shown in Figure 4. This is not surprising due to the redundancy among features observed during correlation analysis. Nevertheless, all feature selection methods seem to agree in neglecting $\rho_{A}$ and $H_{q}$ whereas emphasizing $K$ and $c$.

The preponderance of $K$ can surprise due to the fact that its correlation relationships among features do not significantly differ when overt and covert traffic are compared each other. In any case, such peculiarity does not imply that $K$ cannot be correlated with the class label. But $K$ is indeed not linearly correlated with the class label $(\rho=0.04)$, and univariate statistics in Table 2

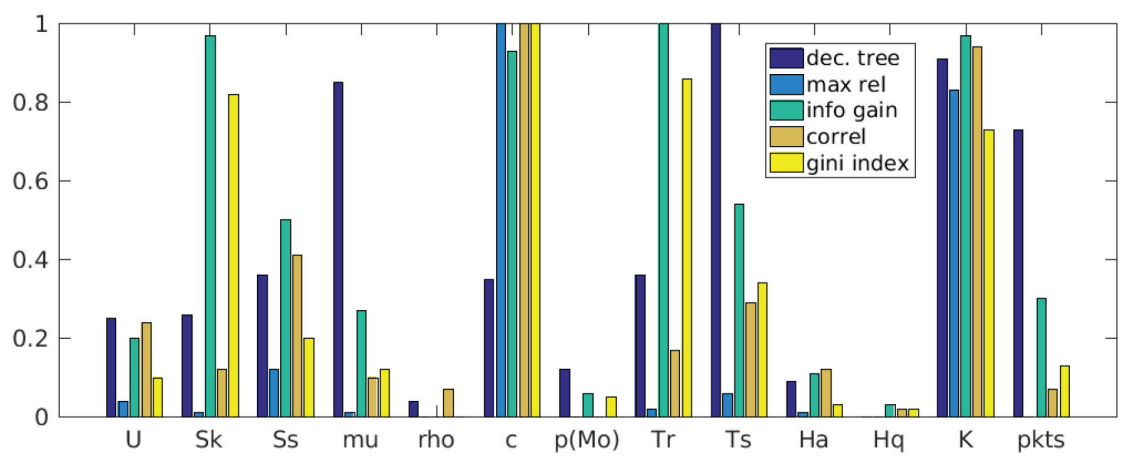

Figure 4 Comparison of feature selection methods. 


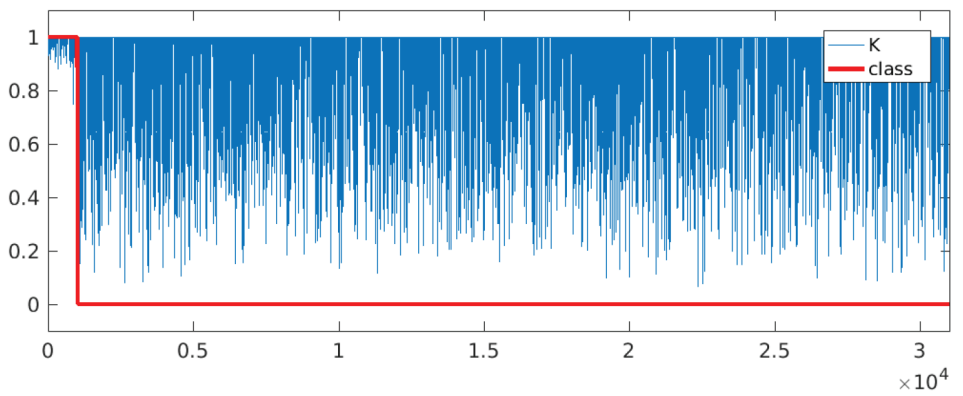

Figure $5 K$ values throughout covert and overt sample flows.

do not show differences between covert and overt traffic for $K$. Nevertheless, $K$ exhibits a noticeable behaviour change when values are visually compared from an overall perspective (Figure 5).

Summarizing, based on the feature selection analysis we can cluster features in four groups, which remark their importance for distinguishing between covert and overt traffic:

- High relevant: $K$ and $c .^{7}$

- Medium relevant: $S_{k}, S_{s}, T_{R}, T_{S}$

- Low relevant: $U, \mu_{\omega S}, H_{a}, p(M o)$, pkts.

- Negligible: $\rho_{A}$ and $H_{q}$.

As observed in [13], where features are selected by decision trees, $c$ is stated as a decisive feature and $\rho_{A}$ is neglected. However, with regard to the previous work the importance of other features vary due to the inclusion of the new regularity and randomness features, derived redundancies and the influence of new feature combinations. Also the selection of overt datasets with longer flows, whose characteristics are more similar to covert flows, has an effect on classifiers and feature selection algorithms, which are forced to give solutions with finer granularity.

\subsection{Binary Classification and Validation}

Based on the feature selection analysis, classification experiments are run to verify the suitability of the selected features. Also, covert channels based on two new techniques are incorporated in a validation step to see if models drawn in training are able to detect novel covert timing techniques, meaning

\footnotetext{
${ }^{7}$ It's worth remembering that $c$ is a function of $S_{k}$ and pkts.
} 
that new covert channels are likely to manipulate traffic capacities in a similar way (from a statistical perspective). Classification is performed with decision tree cores over three different feature sets:

- Feature Set A: high, medium, low and negligible features (all).

- Feature Set B: high, medium and low relevant features.

- Feature Set C: high and medium relevant features.

Tables 3, 4 and 5 show performances for every feature set when decision trees are used to create classification models. The revision of such tables disclose some findings:

- The performance downgrade when using feature set B (high, medium and low relevant) if compared with the feature set $\mathrm{A}$ (all) is minor, likely related to specific non-generalizable cases. From a general perspective $H_{q}$ and $\rho_{A}$ can be considered inefficient for detecting covert timing channels and, therefore, removed.

Table 3 Experiment performance with Feature Set A (all)

\begin{tabular}{|c|c|c|c|c|c|c|}
\hline & \multicolumn{2}{|c|}{ Training } & \multicolumn{2}{|c|}{ Testing } & \multicolumn{2}{|c|}{ Validation } \\
\hline & $\mathrm{PC}$ & $\mathrm{PO}$ & $\mathrm{PC}$ & $\mathrm{PO}$ & $\mathrm{PC}$ & $\mathrm{PO}$ \\
\hline $\mathrm{RC}$ & 1001 (TP) & $21(\mathrm{FN})$ & $986(\mathrm{TP})$ & $50(\mathrm{FN})$ & 1036 (TP) & $196(\mathrm{FN})$ \\
\hline $\mathrm{RO}$ & $40(\mathrm{FP})$ & $29960(\mathrm{TN})$ & $41(\mathrm{FP})$ & $29959(\mathrm{TN})$ & $96(\mathrm{FP})$ & $46097(\mathrm{TN})$ \\
\hline Acc. $(\mathrm{C})$ : & \multicolumn{2}{|c|}{$99.80 \% \pm 0.12 \%$} & \multicolumn{2}{|c|}{$99.71 \%$} & \multicolumn{2}{|c|}{$99.38 \%$} \\
\hline Prec.(C): & \multicolumn{2}{|c|}{$96.20 \% \pm 2.24 \%$} & \multicolumn{2}{|c|}{$96.01 \%$} & \multicolumn{2}{|c|}{$91.52 \%$} \\
\hline $\operatorname{Recall}(\mathrm{C})$ : & \multicolumn{2}{|c|}{$97.95 \% \pm 1.75 \%$} & \multicolumn{2}{|c|}{$95.17 \%$} & \multicolumn{2}{|c|}{$84.09 \%$} \\
\hline $\operatorname{AUC}(C):$ & \multicolumn{2}{|c|}{$0.962 \pm 0.099$} & \multicolumn{2}{|c|}{0.973} & \multicolumn{2}{|c|}{0.903} \\
\hline
\end{tabular}

PC: predicted covert, PO: predicted overt, RC: real covert, RO: real overt, TP: true positive, TN: true negative, FP: false positive, FN: false negative,

(C): covert as positive class, Acc.: accuracy, Prec.: precision, AUC: area under ROC curve.

Table 4 Experiment performance with Feature Set B (excluded negligible)

\begin{tabular}{|c|c|c|c|c|c|c|}
\hline & \multicolumn{2}{|c|}{ Training } & \multicolumn{2}{|c|}{ Testing } & \multicolumn{2}{|c|}{ Validation } \\
\hline & $\mathrm{PC}$ & $\mathrm{PO}$ & PC & $\mathrm{PO}$ & PC & $\mathrm{PO}$ \\
\hline $\mathrm{RC}$ & 997 (TP) & $25(\mathrm{FN})$ & $946(\mathrm{TP})$ & $74(\mathrm{FN})$ & 991 (TP) & $241(\mathrm{FN})$ \\
\hline RO & 37 (FP) & $29963(\mathrm{TN})$ & $96(\mathrm{FP})$ & $29961(\mathrm{TN})$ & $81(\mathrm{FP})$ & $46112(\mathrm{TN})$ \\
\hline$\overline{\text { Acc. }(\mathrm{C}) \text { : }}$ & \multicolumn{2}{|c|}{$99.80 \% \pm 0.08 \%$} & \multicolumn{2}{|c|}{$99.54 \%$} & \multicolumn{2}{|c|}{$99.32 \%$} \\
\hline Prec.(C): & \multicolumn{2}{|c|}{$96.45 \% \pm 1.65 \%$} & \multicolumn{2}{|c|}{$96.10 \%$} & \multicolumn{2}{|c|}{$92.44 \%$} \\
\hline Recall(C): & \multicolumn{2}{|c|}{$97.56 \% \pm 1.60 \%$} & \multicolumn{2}{|c|}{$92.86 \%$} & \multicolumn{2}{|c|}{$80.44 \%$} \\
\hline $\operatorname{AUC}(C):$ & \multicolumn{2}{|c|}{$0.959 \pm 0.100$} & \multicolumn{2}{|c|}{0.973} & \multicolumn{2}{|c|}{0.924} \\
\hline
\end{tabular}

PC: predicted covert, PO: predicted overt, RC: real covert, RO: real overt, TP: true positive, TN: true negative, FP: false positive, FN: false negative,

(C): covert as positive class, Acc.: accuracy, Prec.: precision, AUC: area under ROC curve. 
Table 5 Experiment performance with Feature Set C (excluded negligible and low relevant)

\begin{tabular}{|c|c|c|c|c|c|c|}
\hline & \multicolumn{2}{|c|}{ Training } & \multicolumn{2}{|c|}{ Testing } & \multicolumn{2}{|c|}{ Validation } \\
\hline & $\mathrm{PC}$ & $\mathrm{PO}$ & $\mathrm{PC}$ & $\mathrm{PO}$ & $\mathrm{PC}$ & $\mathrm{PO}$ \\
\hline $\mathrm{RC}$ & 893 (TP) & $129(\mathrm{FN})$ & 915 (TP) & $121(\mathrm{FN})$ & 980 (TP) & $252(\mathrm{FN})$ \\
\hline RO & $57(\mathrm{FP})$ & 29943 (TN) & $65(\mathrm{FP})$ & $29935(\mathrm{TN})$ & $142(\mathrm{FP})$ & $46051(\mathrm{TN})$ \\
\hline Acc.(C): & \multicolumn{2}{|c|}{$99.40 \% \pm 0.21 \%$} & \multicolumn{2}{|c|}{$99.17 \%$} & \multicolumn{2}{|c|}{$99.30 \%$} \\
\hline Prec.(C): & \multicolumn{2}{|c|}{$94.23 \% \pm 2.74 \%$} & \multicolumn{2}{|c|}{$93.37 \%$} & \multicolumn{2}{|c|}{$87.34 \%$} \\
\hline $\operatorname{Recall}(\mathrm{C})$ : & \multicolumn{2}{|c|}{$87.39 \% \pm 8.07 \%$} & \multicolumn{2}{|c|}{$88.32 \%$} & \multicolumn{2}{|c|}{$79.55 \%$} \\
\hline $\operatorname{AUC}(\mathrm{C}):$ & \multicolumn{2}{|c|}{$0.931 \pm 0.043$} & \multicolumn{2}{|c|}{0.920} & \multicolumn{2}{|c|}{0.824} \\
\hline
\end{tabular}

PC: predicted covert, PO: predicted overt, RC: real covert, RO: real overt, TP: true positive, TN: true negative, FP: false positive, FN: false negative,

(C): covert as positive class, Acc.: accuracy, Prec.: precision, AUC: area under ROC curve.

- The performance downgrade when using feature set $\mathrm{C}$ (high and medium relevant) if compared with the feature set A or B is considerable. Therefore, low relevant features- $\mu_{\omega S}, H_{a}, p(M o)$, pkts-are still determinant for the classification and should be included regardless of possible redundancies.

- The downgrade in the validation phase is obvious in spite of the used feature set. False negatives mainly belong to the novel not-trained techniques ED1 and ED2 (Table 6), meaning that new techniques have room to exploit channel capacities in ways that can bypass classifiers trained with known, old techniques.

Similar test have been carried out with other classifiers, specifically: random forests, SVM, neural networks, Bayes-based ensemble and k-Nearest

Table 6 Identification of FN and FP in the validation phase for the relevant feature set

\begin{tabular}{lccc}
\hline Technique & Wrong & $\mu_{\text {conf }}(0)$ & $\mu_{\text {conf }}(1)$ \\
\hline CAB (FN) & 8 & $1.00 \pm 0.00$ & $0.00 \pm 0.00$ \\
BER (FN) & 18 & $1.00 \pm 0.00$ & $0.00 \pm 0.00$ \\
SHA (FN) & 2 & $0.75 \pm 0.35$ & $0.25 \pm 0.35$ \\
GAS (FN) & 24 & $1.00 \pm 0.00$ & $0.00 \pm 0.00$ \\
JIN (FN) & 12 & $1.00 \pm 0.00$ & $0.00 \pm 0.00$ \\
LUO (FN) & 8 & $1.00 \pm 0.00$ & $0.00 \pm 0.00$ \\
ZAN (FN) & 11 & $1.00 \pm 0.00$ & $0.00 \pm 0.00$ \\
GIF (FN) & 3 & $0.78 \pm 0.19$ & $0.22 \pm 0.19$ \\
ED1 (FN) & 99 & $1.00 \pm 0.00$ & $0.00 \pm 0.00$ \\
ED2 (FN) & 55 & $0.70 \pm 0.10$ & $0.30 \pm 0.10$ \\
\hline Overt (FP) & 81 & $0.05 \pm 0.09$ & $0.05 \pm 0.09$
\end{tabular}

FP: false positive, FN: false negative, wrong: total number of wrong classified flows $\mu_{\text {conf }}(0)$ and $\mu_{\text {conf }}(1)$ stand respectively for the average decision tree confidence to establish the 0 (overt) or a 1 (covert) label to the misclassified samples. 
neighbors learners. A plain decision tree obtained the best performances ${ }^{8}$, yet the previous discussed aspects are common in all tested learning schemes.

\subsection{Unsupervised Analysis}

Finally, tests by outlier ranking algorithms tried to elucidate if the new considered features could provide extra information to face the detection of covert timing channels from an unsupervised manner. Experiments in [15] revealed that covert timing channels can hardly be seen as outliers. Here, outlier analysis are performed by considering two different feature sets:

- Feature Set B: high, medium and low relevant features.

- Feature Set D: new high, medium and low relevant features not used in [15], i.e., $T_{R}, T_{S}, H_{a}$ and $K$.

Results are shown in Table 7 and Figure 6. Results confirm that density-based outlier detection is completely useless for detecting covert timing channels. Only the HBOS algorithm (histogram-based method) can face the task to differentiate between overt and covert flows. However, the probabilities to suspect that a legitimate flow is a covert flow are still too high. For example, the P@n index gives the proportion of correct results in the top $n$ ranks.

Table 7 Outlier detection performance indices for feature set B and D

\begin{tabular}{|c|c|c|c|c|c|c|c|}
\hline \multicolumn{8}{|c|}{ Feature set B } \\
\hline & $\mathrm{P} @ \mathrm{n}$ & Adj.P@n & AP & Adj. AP & MaxF1 & Adj.MaxF1 & ROC/AUC \\
\hline$\overline{\mathrm{LOF}}$ & 0.02 & -0.01 & 0.01 & -0.03 & 0.08 & 0.05 & 0.55 \\
\hline $\mathrm{COF}$ & 0.01 & -0.02 & 0.00 & -0.03 & 0.07 & 0.04 & 0.53 \\
\hline INFLO & 0.02 & -0.01 & 0.01 & -0.03 & 0.08 & 0.05 & 0.51 \\
\hline HBOS & 0.28 & 0.26 & 0.27 & 0.25 & 0.49 & 0.47 & 0.96 \\
\hline \multicolumn{8}{|c|}{ Feature set D } \\
\hline & $\mathrm{P} @ \mathrm{n}$ & Adj.P@n & AP & Adj. AP & MaxF1 & Adj.MaxF1 & ROC/AUC \\
\hline$\overline{\mathrm{LOF}}$ & 0.04 & 0.01 & 0.02 & -0.01 & 0.08 & 0.05 & 0.57 \\
\hline $\mathrm{COF}$ & 0.00 & -0.03 & 0.00 & -0.03 & 0.09 & 0.06 & 0.62 \\
\hline INFLO & 0.03 & 0.00 & 0.02 & -0.01 & 0.07 & 0.04 & 0.52 \\
\hline HBOS & 0.09 & 0.06 & 0.12 & 0.10 & 0.36 & 0.34 & 0.92 \\
\hline
\end{tabular}

\footnotetext{
${ }^{8}$ The decision tree configuration included pre- and postpruning to avoid overfitting and favor generalization. It used Information Gain (i.e., entropy-based) as splitting criterion; the minimal size for splitting was four samples; the minimal leaf size was two samples, allowing a maximal tree depth of 20 levels; the minimal gain for splitting a node was 0.1 ; the confidence level used for the pessimistic error calculation of pruning was 0.25 , whereas the number of prepruning alternatives was three. In addition, a 10-fold cross-validation process was performed to reinforce disclosed models.
} 

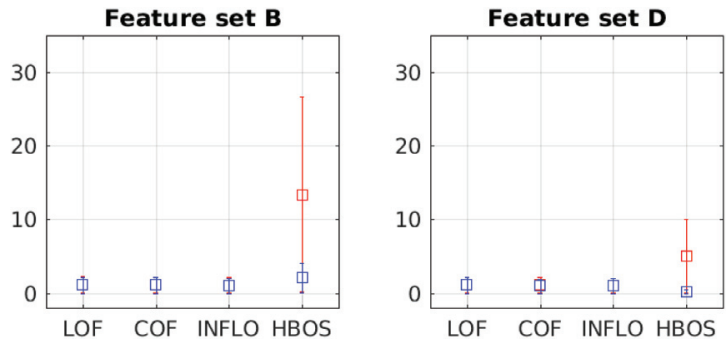

Figure 6 Outlier ranking results. Again, distributions are not normal but strongly skewed. Plots show medians and Confidence Intervals over the median (approximately 95\%). Red markers correspond to covert datasets, blue for overt datasets. Red lines are indistinguishable (or almost) in LOF, COF and INFLO due to value overlap.

$\mathrm{P} @ \mathrm{n}=0.28$ in Table 7 is the best result for the HBOS case, but still too low to build a practical detector. AUC rates are nevertheless significantly high for HBOS. The AUC index can be understood as the probability that the algorithm ranks a randomly chosen positive example higher than a randomly chosen negative example. Summarizing, results show that practically all flows with covert channels obtain high HBOS ranks; overt flows are low-ranked instead, but still some overt flows-a meaningless proportion but still many when compared with covert flows rates-score high and even higher than covert cases.

Already in [15] HBOS was the only method to find a significant difference between overt and covert flows. The inclusion of the new features $\left(T_{R}, T_{S}, H_{a}\right.$ and $K$ ) reinforces the outlier nature of flows with covert channels, as shown in Figure 6, but is still far from being discriminant.

Therefore, the main findings of the unsupervised experiments are:

- As a general trend, covert channel flows obtain higher outlierness ranks when analyzed by the HBOS algorithm (histogram, distance-based), but still a significant proportion of overt flows scores equally high or even higher.

- The new features $T_{R}, T_{S}, H_{a}$ and $K$ endorse the outlier nature of covert channels, but the most revealing outlierness ranks are obtained when they are combined and used together with $S_{k}, S_{s}, c, U, \mu_{\omega S}, p(M o)$ and pkts.

\section{Conclusions}

In this work we have deeply analyzed the capacity of some network traffic features to disclose covert timing channels. All analyzed features are measured or calculated from network traffic flows IATs (Inter Arrival Times). 
From the analyzed features, the most relevant ones for the detection of covert channels are: $c, S_{k}$, pkts and $K . c$ is the estimation of potential covert byte-equivalent symbols, which depends on the number of packets in the flow (pkts) and the number of multimodes that appear in the probability density estimation when using gaussians as kernels $\left(S_{k}\right) . K$ is the Kolmogorov complexity estimation approximated by using zlib compression. On the other hand, the hurst coefficient $\left(H_{q}\right)$ and autocorrelation-based coeffcients $\left(\rho_{A}\right)$ are found inefficient and can be discarded.

Conducted experiments show that the detection of covert timing channels is a demanding challenge and new covert techniques can easily bypass trained detection schemes. The high variety of forms that network traffic can take and the extremely low expected rate of covert channels in real traffic are the main reasons that make the accurate detection so difficult. Combination of supervised and unsupervised techniques (i.e., semi-supervise methods) appears as the right direction to follow in order to develop satisfactory detectors. However, a considerable rate of false positives is almost unavoidable unless more complex methods are developed and implemented.

\section{Acknowledgment}

The research leading to these results has been partially funded by the Vienna Science and Technology Fund (WWTF) through project ICT15-129, BigDAMA.

\section{References}

[1] V. Berk, A. Giani, G. Cybenko, and N. Hanover (2005). Detection of covert channel encoding in network packet delays. Rapport technique TR536, de lUniversite de Dartmouth, 19.

[2] James V. Bradley (1968). Distribution-Free Statistical Tests, 1st Edition. Prentice-Hall.

[3] M. Breunig, H.-P. Kriegel, R. T. Ng, and J. Sander (2000). Lof: Identifying density-based local outliers. SIGMOD Rec. 29, 93-104.

[4] S. Cabuk, C. E. Brodley, and C. Shields (2004). IP covert timing channels: Design and detection. In Proceedings of the 11th ACM Conference on Computer and Communications Security, CCS'04, (ACM: New York, NY, USA), 178-187.

[5] G. O. Campos, A. Zimek, J. Sander, R. J. G. B. Campello, B. Micenkov, E. Schubert, I. Assent, and M. E. Houle (2016). On the evaluation of 
unsupervised outlier detection: Measures, datasets, and an empirical study. Data Mining and Knowledge Discovery, 30, 891-927.

[6] A. Carbone, G. Castelli, and H. E. Stanley (2004). Time-dependent hurst exponent in financial time series. Applications of Physics in Financial Analysis 4 (APFA4). Physica A: Statistical Mechanics and Its Applications, 344, 267-271.

[7] E. J. Castillo, X. Mountrouidou, and X. Li (2017). "Time Lord: Covert Timing Channel Implementation and Realistic Experimentation," inProceedings of the 2017 ACM SIGCSE Technical Symposium on Computer Science Education, SIGCSE'17, (ACM: New York, NY, USA), $755-756$.

[8] ElevenPaths. Low cost malware that uses gmail as a covert channel. Telefonica Digital España, Technical report, May 2016.

[9] W. Gasior and L. Yang (2011). "Network covert channels on the android platform," in Proceedings of the Seventh Annual Workshop on Cyber Security and Information Intelligence Research, CSIIRW'11, (ACM: New York, NY, USA), p. 61:1.

[10] J. Giffin, R. Greenstadt, P. Litwack, and R. Tibbetts (2003). Covert Messaging through TCP Timestamps. Springer: Berlin, Heidelberg, 194-208.

[11] M. Goldstein and A. Dengel (2012). "Histogram-based Outlier Score (HBOS): A Fast Unsupervised Anomaly Detection Algorithm," in Advances in Artificial Intelligence, (KI-2012), ed. S. Wölfl, pp. 59-63. [Online, 9, 2012].

[12] F. Iglesias, R. Annessi, and T. Zseby (2016). DAT detectors: uncovering TCP/IP covert channels by descriptive analytics. Security and Communication Networks, 9, 3011-3029.

[13] F. Iglesias, V. Bernhardt, R. Annessi, and T. Zseby (2017). "Decision tree rule induction for detecting covert timing channels in TCP/IP traffic," in First IFIP TC 5, WG 8.4, 8.9, 12.9 International Cross-Domain Conference, CD-MAKE 2017, in (eds) Holzinger A., Kieseberg P., Tjoa A., Weippl E., Lecture Notes in Computer Science, Vol. 10410, ( Springer: Cham), 105-122.

[14] F. Iglesias and T. Zseby (2015). Analysis of network traffic features for anomaly detection. Machine Learning, 101, 59-84.

[15] F. Iglesias and T. Zseby (2017). "Are network covert timing channels statistical anomalies?" in Proceedings of the 12th International Conference on Availability, Reliability and Security, ARES'17, (ACM: New York, NY, USA), 81:1-81:9. 
[16] W. Jin, A. K. H. Tung, J. Han, and W. Wang (2006). Ranking Outliers Using Symmetric Neighborhood Relationship. Springer: Berlin Heidelberg, 577-593.

[17] T. Kleinow (2002). Testing continuous time models in financial markets. $\mathrm{PhD}$ thesis, Humboldt-Universitát zu Berlin, Wirtschaftswissenschaftliche Fakultt, 2002.

[18] A. N. Kolmogorov (1968). Three approaches to the quantitative definition of information. International Journal of Computer Mathematics, 2, 157168.

[19] M. Li and P. Vitanyi (1997). An Introduction to Kolmogorov Complexity and Its Applications (Texts in Computer Science). Springer.

[20] X. Luo, E. W. W. Chan, and R. K. C. Chang (2008). "TCP covert timing channels: Design and detection," in IEEE International Conference on Dependable Systems and Networks with FTCS and DCC (DSN), 420429.

[21] Henry B. Mann (1945). On a test for randomness based on signs of differences. Ann. Math. Statist. 16, 193-199.

[22] N. Meinshausen and P. Bhlmann (2010). Stability selection. Journal of the Royal Statistical Society: Series B (Statistical Methodology), 72, 417473.

[23] B. H. Menze, B. M. Kelm, R. Masuch, U. Himmelreich, P. Bachert, W. Petrich, and and F. A. Hamprecht (2009). A comparison of random forest and its Gini importance with standard chemometric methods for the feature selection and classification of spectral data. BMC Bioinformatics, $10,213$.

[24] MEJ Newman (2005). Power laws, Pareto distributions and Zipf's law. Contemporary Physics, 46, 323-351.

[25] M. A. Padlipsky, D. W. Snow, and P. A. Karger (1978). Limitations of end-to-end encryption in secure computer networks, 1978. ESD-TR-78158.

[26] H. Peng, F. Long, and C. Ding (2005). Feature selection based on mutual in- formation criteria of max-dependency, max-relevance, and min-redundancy. IEEE Transactions on Pattern Analysis and Machine Intelligence, 27, 1226-1238.

[27] S. M. Pincus (1991). Approximate entropy as a measure of system complexity. Proceedings of the National Academy of Sciences, 88, 2297-2301. 
[28] G. Shah, A. Molina, and M. Blaze (2006). "Keyboards and covert channels," in Proceedings of the 15th Conference on USENIX Security Symposium, USENIX-SS'06, USENIX Association: Berkeley, CA, USA.

[29] B. W. Silverman. Using kernel density estimates to investigate multimodality. Journal of the Royal Statistical Society: Series B, 43, 97-99.

[30] J. Tang, Z. Chen, A. W. Fu, and D. W.-L. Cheung (2002). "Enhancing effectiveness of outlier detections for low density patterns," in Proceedings of the 6th Pacific-Asia Conference on Advances in Knowledge Discovery and Data Mining, PAKDD'02, (Springer-Verlag: London, UK), 535-548.

[31] TU Wien CN Group. Data Analysis and Algorithms, 2017.

[32] J. Wu, Y. Wang, L. Ding, and X. Liao (2012). Improving performance of network covert timing channel through Huffman coding. Mathematical and Computer Modelling, 55, 69-79, 2012.

[33] S. Zander, G. Armitage, and P. Branch (2007). "An empirical evaluation of ip time to live covert channels," in 2007 15th IEEE International Conference on Networks, ICON 2007, (IEEE), 42-47.

\section{Biographies}

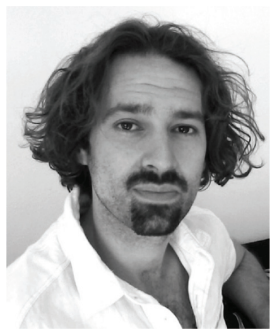

Félix Iglesias Vázquez was born in Madrid, Spain, in 1980. He obtained the Dipl.-Ing. in electrical engineering and MAS in IT from the Ramon Llull University, Barcelona, Spain. In 2012 he received the Ph.D. degree in technical sciences from TU Wien, Austria, where he currently holds a University Assistant position doing fundamental research in data analysis and network security. He has worked on R\&D for diverse Spanish and Austrian firms, and lectured in the fields of electronics, physics, automation, machine learning and data analysis. 


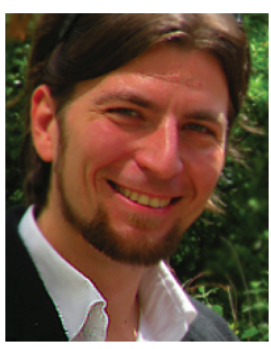

Robert Annessi received his B.Sc. and his M.Sc. degrees in computer engineering from TU Wien in 2011 and 2014 respectively. He is genuinely interested in communication networks, network security, and privacy, and is currently pursuing his Ph.D in the area of secure group communication for critical infrastructures. His further research interests are anonymous communication, covert communication, and subliminal communication.

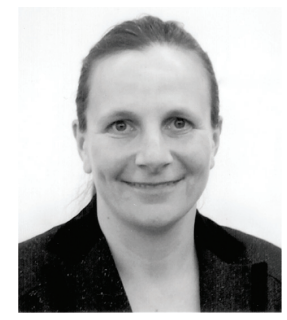

Tanja Zseby is a professor of communication networks in the Faculty of Electrical Engineering and Information Technology at TU Wien. She received her Dipl.-Ing. degree in electrical engineering and her Ph.D. (Dr.-Ing.) from Technical University Berlin, Germany. Before joining TU Wien she led the Competence Center for Network Research at the Fraunhofer Institute for Open Communication Systems (FOKUS) in Berlin and worked as visiting scientist at the University of California, San Diego. 